\title{
Microcantilever-Based Nanomechanical Studies of the Orphan Nuclear Receptor Pregnane X Receptor-Ligand Interactions
}

\author{
Kasey L. Hill, Pampa Dutta, Zhou Long, Michael J. Sepaniak \\ Department of Chemistry, University of Tennessee, Knoxville, USA. \\ Email: msepaniak@utk.edu \\ Received September $10^{\text {th }}, 2010$; revised January $1^{\text {st }}, 2010$; accepted January $5^{\text {th }}, 2011$.
}

\begin{abstract}
Human pregnane $X$ receptor $(P X R)$ is of vital importance in pharmaceutical and exogenous compound metabolism within the body. This provides strong motivation for investigating this orphan receptor's activation by various pharmaceuticals, xenobiotics, and endocrine disrupting chemicals (EDCs). A nanomechanical transducer is developed to study xenobiotic and EDC interactions with the bioreceptor PXR's ligand binding domain (LBD). The combination of immobilized LBD PXR with a nanostructured microcantilever (MC) platform allows for the sensitive, label-free study of ligand interaction with the receptor. PXR shows real-time, reversible responses when exposed to a specific pharmaceutical, EDCs, and xenobiotic ligands. Three EDCs binding interactions are tested, which include phthalic acid, nonylphenol, and bisphenol A, with PXR. PXR LBD was exposed to rifampicin, a potent PXR activator, with various injection and recovery times to study their interaction. A two protein array of PXR and estrogen receptor $\alpha(E R-\alpha)$ directly compares the nanomechanical responses of these receptors with rifampicin on a single platform.
\end{abstract}

Keywords: Microcantilevers, Nanobiosensing, Pregnane X Receptor, Estrogen Receptor, Endocrine Disrupting Chemicals, Bioarray

\section{Introduction}

Humans are exposed to harmful chemicals and contaminants each day. It is essential that these toxins are removed or detoxed from the body. These foreign compounds or xenobiotics, which include environmental toxins, endogenous hormones, steroids, pharmaceuticals, and dietary supplements, trigger a line of defense mechanisms within the body. The family of cytochrome P450 enzymes are the main xenobiotic defenders for mammals. These enzymes include four families of Cytochrome P450 monooxygenases: CYP1, CYP2, CYP3, and CYP4 [1]. Cytochrome P4503A4 (CYP3A4), a critical member of our defense system, makes the removal of many unwanted xenobiotics possible [2]. CYP3A4 and its isoforms are highly involved in pharmaceutical metabolism, playing a significant role in metabolizing approximately $50 \%$ of drugs used today $[3,4]$. When xenobiotic ligands bind to a specific nuclear hormone receptor, the interaction transcriptionally activates CYP3A4 [5]. In 1998, a novel orphan human nuclear receptor was identified and termed pregnane $\mathrm{X}$ receptor
(PXR), steroid and xenobiotic receptor (SXR), pregnane activated receptor (PAR), and NR1I2 [6-9]. Herein, we choose to use the term PXR.

PXR is activated by a broad array of structurally diverse xenobiotics [10]. This wide activation range is possible is due to a unique ligand binding domain (LBD) or pocket, which can expand to fit a variety of sized ligands. PXR's LBD has two $\beta$ strands that are not present in other nuclear receptors and that allow its expansion [11]. This flexible, hydrophobic LBD allows PXR to be activated by a diverse range of synthetic and naturally occurring chemicals making it an interesting candidate to serve as a xenobiotic sensor [12].

Importantly, PXR is activated by numerous pharmaceuticals with diverse properties, functions, and structures and controls the expression of genes that are vital to pharmaceutical metaolism $[13,14]$. PXR activation mediates transcription of CYP enzymes, which are considered drug metabolizing enzymes [1,12]. Since PXR activation allows for regulation and expression of CYP3A this interaction is critical to drug metabolism [2]. Pharmaceutical metabolism and interaction is vital to monitor and 
prevent drug-drug interactions. Drug-drug interactions can occur when co-administered drugs alter the efficacy of one another. This usually occurs when one drug increases or decreases the metabolism of another $[3,14]$. Contaminants could modify drug concentrations in vivo and skew the prescribed therapeutic dose.

High throughput, sensitive screening and detection of xenobiotics are critical to determine harmful toxins and possible drug-drug interactions. A highly sensitive, inexpensive, and rapid sensor utilizing the nuclear receptor PXR has been developed and is demonstrated within. Recently, biosensors based on microcantilevers (MCs) have been used to detect and screen for many harmful environmental contaminants using estrogen and thyroid receptor proteins [16,17]. The high sensitivity and widespread availability of inexpensive MCs has generated intense interest in their use as chemical and biological sensors [18-27]. Additionally, MCs can be used with on-chip circuitry and in microcantilever arrays for high throughput and simultaneous differential assays and bioaffinity studies with a very small transducer footprint that potentially could be employed in the field.

A MC suitable for biosensing is modified on one side with a nanostructured layer and a receptor phase that has some degree of affinity for the analyte. By exploiting PXR's affinity for a diverse range of ligands, we are able to screen for xenobiotics quickly and without extensive, time-consuming labeling techniques and cell preparation $[10,14,15,28,29]$. Specific interactions of the target analytes with the receptor can cause an apparent surface stress and nanomechanical bending that may be conveniently monitored based on the beam bending technique commonly used in atomic force microscopy. The static bending (tip deflection, $z_{\max }$ ) of the MC varies in selectivity and sensitivity due to preferential binding of analyte molecules on the functionalized, active MC surface and is governed by Stoney's equation [30]

$$
z_{\max }=\frac{3 l^{2}(1-v)}{E t^{2}} \Delta \sigma
$$

where $v$ and $\mathrm{E}$ are, respectively, the Poisson ratio and Young's modulus for the cantilever, $t$ is the thickness of the MC, $l$ is the cantilever effective length, and $\Delta \sigma$ is analyte-induced differential surface stress $\left(\Delta \sigma_{\text {active side }}\right.$ $\Delta \sigma_{\text {passive side) }}$.

We demonstrate that detection and screening for pharmaceuticals and endocrine disrupting chemicals (EDCs) can be accomplished with functionalized MCs. These sensors provide real-time measurements of surface stress changes resulting from ligand interaction with immobilized proteins in the low-to-sub-nanomolar range [20]. Sensitivity is critical in biosensors due to the ultra-trace concentrations of many xenobiotics that can impact biological systems. Nanostructured MC biosensors allow detection without labels and in a detection range that is applicable for real biological systems [31]. PXR immobilized on a nanostructured MC surface provides sensitive and reversible detection of various pharmaceuticals and environmental contaminants or EDCs. To our knowledge, this is the first time the orphan nuclear receptor PXR has been immobilized on a MC surface. Due to PXR's unique nature we saw interesting results in our concentration studies with rifampicin as well as surprising responses when utilizing differently tagged PXR-receptors.

\section{Materials and Methods}

\subsection{Reagents}

Experiments were performed using commercially available silicon arrays of MCs having dimensions $400 \mu \mathrm{m}$ length, $100 \mu \mathrm{m}$ width, and approximately $1 \mu \mathrm{m}$ thick (Mikro Masch Co., Sunnyvale, CA). Chromium, gold, and silver metals deposited on the MCs were obtained from Kurt J. Lesker, Gatewest, and Alfa Aesar Co., respectively, at $99.9 \%$ purity. 2-aminoethanethiolhydrochloride (AET), glutaraldehyde (GA), the salts employed for the preparation of buffer solutions, and all other reagents were purchased from Sigma-Aldrich Chemical Co.(St. Louis, MO) or Fisher Scientific at highest available purity and used as received. The test ligands rifampicin, pregnenolone-16 $\alpha$-carbonitrile (PCN), 3-methyl-cholanthrene (3-MC), phthalic acid, nonylphenol and bisphenol A were also obtained from Sigma-Aldrich. Glutathiones-transferase (GST)-tagged human PXR, alexa fluor 633 anti-IgG, and estrogen receptor $\alpha$ (ER- $\alpha$ ) were purchased from Invitrogen (Carlsbad, California). 6-histidine (6HIS)-tagged human PXR was generously provided by Astra Zeneca. Ovalbumin and FITC-anti-IgG was purchased from Sigma-Aldrich. Water used to prepare solutions was obtained from a Branstead E-pure water filtration system.

\subsection{Cantilever Modification}

The process of preparing and creating nanostructured surfaces on MCs is described in detail elsewhere [32].

The MCs were placed into a physical vapor deposition chamber (Cooke Vacuum Products, Model CVE 301, South Norwalk, CT) to be coated on one side with the appropriate metallic films using thermal deposition. To create a nanostructured MC, a thin film ( $\sim 5 \mathrm{~nm}$ ) of chromium was applied to the surface to act as an adhesion layer followed by a thin film of gold ( $15 \mathrm{~nm})$. Next, a film consisting of gold and silver was co-depo-sited. Subsequently, the silver was chemically 
removed via oxidation from the film ("dealloying") using an aqueous solution of $5 \mathrm{mg} / \mathrm{mL} \mathrm{HAuCl}_{4}$ leaving a gold surface with nanosized, colloid-like features. The thick-ness of the dealloyed gold layer was $\sim 100$ or $\sim 150 \mathrm{~nm}$ in these studies.

Nanostructured MCs were chemically modified for protein immobilization by the process detailed elsewhere [17]. MCs were immersed in $1 \mathrm{mM}$ aqueous solution of AET for one hour producing a self-assembled monolayer on the cantilever surface. Following thorough rinsing in deionized water, the amino groups were derivatized with the cross linker by immersing the cantilever in a $1 \%(\mathrm{w} / \mathrm{v})$ solution of GA in $10 \mathrm{mM}$ phosphate buffered saline (PBS), pH 8.0 for three hours [33,34]. Subsequently, immobilization of both the PXR LBD nuclear receptor and the ovalbumin was achieved in random orientation by dipping the functionalized cantilevers into $100 \mathrm{mg} / \mathrm{L}$ solutions of protein in $10 \mathrm{mM}$ PBS, $\mathrm{pH} 7.0$ for four hours at $4^{\circ} \mathrm{C}$. Although we used an array of MCs, in this study we chemically treated all the cantilevers the same where both PXR and ovalbumin were separately immobilized on the functionalized surfaces of different cantilevers from separate arrays and a single randomly chosen MC within an array response was recorded. For the two protein array, the immobilization of both the PXR and the ER- $\alpha$ was achieved in random orientation utilizing a capillary coating method described in detail elsewhere [35]. Each capillary contained $100 \mathrm{mg} / \mathrm{L}$ solution of receptor in $10 \mathrm{mM}$ PBS, pH 7.0, which was placed over each lever for 1 hour at room temperature.

\subsection{Instrumentation}

The MC deflection measurements were carried out using the optical beam-deflection technique described elsewhere $[16,17]$. The apparatus similar to that depicted in Figure 1 included a $5 \mathrm{~mW} 632 \mathrm{~nm}$ diode laser (Coherent Laser Corp., Auburn, CA), a spatial filtering and focusing system, and an in-house built position sensitive optical detector. The output of the detector was displayed and recorded using a SRS 850 DSP lock-in amplifier as a multichannel digital recorder (Stanford Research Systems, Sunnyvale, CA). The signal output is recorded as volts (approximately $1 \mathrm{~nm} \mathrm{z}_{\max }$ per $\mathrm{mV}$ output). Data was collected at $1 \mathrm{~Hz}$ and then a moving averaging algorithm covering 180 data points was used to generate the figures presented herein. This smoothing did not alter the shape of the true response curves [19].

The cantilever system was mounted inside a $\sim 5 \mu \mathrm{L}$ volume flow cell built in house previously described elsewhere [16]. A Watec CCD camera (Edmund Indust- rial Optics, Barrington, NJ) was used to image the MC chip in the flow cell and facilitate in aligning the focused laser

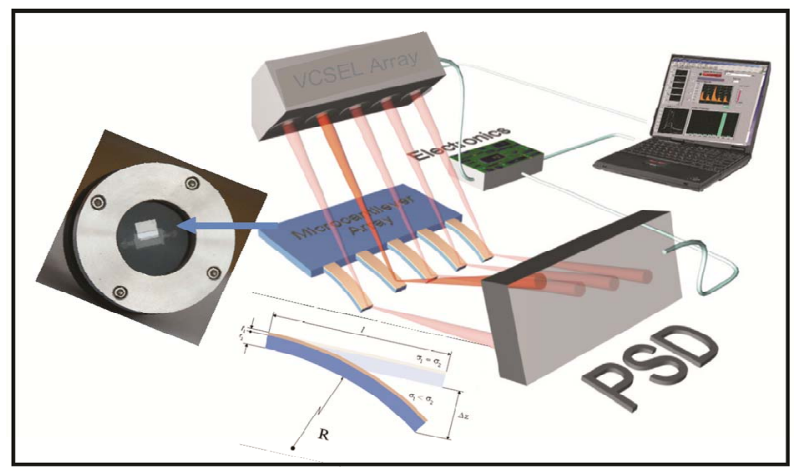

Figure 1. Schematic of laser array setup with a single PSD. Chip array within flow cell is shown. The lower left shows bending of a single MC due to adsorption-induced differential stress. Note that deformation of the MC is characterized by the radius of curvature, $R$, and tip deflection, $\otimes z$, according to Equation (1).

beam to reflect off the cantilever tip. Analyte solutions were delivered to the flow cell via a system of vessels connected to three-way valves allowing for switching between different solutions. The gravity-driven flow was generally adjusted to $100 \mu \mathrm{L} /$ minute by adjusting vessel height.

Many of the pharmaceuticals and EDCs are sparingly soluble in water. Thus, $10 \mathrm{mM}$ stock solutions of all EDCs and some pharmaceuticals were prepared in pure methanol and then diluted with $10 \mathrm{mM}$ PBS, pH 7.0 to make the desired concentration of each analyte [Caution: because of their potential harmful effects, care must be taken in the handling and disposing of EDC and pharmaceutical solutions]. PCN pharmaceutical stock solution was prepared in acetone then diluted with PBS. PBS was also used as a background solution. MCs mounted in the flow cell were initially allowed to equilibrate in PBS until the signal was stable. For our purposes, tensile and compressive responses involve contraction and expansion of the active MC surface, respectively.

MC deflection measurements of single protein functionalized chips using a single diode laser or multiple protein arrays can be monitored using an array of vertical cavity surface emitting lasers (VCSELs) which are focused onto the tip of each MC (Figure 1). The reflected beam is captured and monitored by a single position sensitive detector as depicted in the figure. Although in the work reported herein employed a single laser setup, we often use a VCSEL setup [36-38].

\section{Results and Discussion}

Our studies focus on developing MC systems utilizing the nuclear receptor PXR as the immobilized bioreceptor 
phase. We demonstrate that our immobilization process does not appreciably denature the PXR. The magnitude based response order for PXR activators is maintained when compared to well-established assays. With these PXR active analytes, PXR selectivity is demonstrated when compared to a similarly sized immobilized protein. The assay magnitude based response order is maintained when PXR is exposed to EDCs. Reproducibility and sensitivity is illustrated using a potent PXR activator, rifampicin. A two protein array is developed to compare PXR and ER- $\alpha$ interaction with rifampicin.

Sensitivity enhancement in biosensing is key due to the small concentrations of ligands that can activate nuclear receptors. By nanostructuring the active MC surface, we are able to substantially improve the sensitivity, which has in many cases surpassed the increase in surface area $[18,32,39]$. The initial "dealloying" of the MC surface provides a greater surface area for nuclear receptor immobilization, which leads to an enhancement in sensitivity. Our developing PXR MC biosensor, in support of fundamental nuclear receptor studies, may prove to be a quick, less expensive method for EDC screening and possible drug-drug interaction predictions.

\subsection{Detection of Known PXR Activators and EDCs Using PXR Modified MCs}

Figure 2 compares the responses of a PXR functionalized MC when exposed to three analytes in PBS. This figure illustrates that $1.0 \mu \mathrm{M}$ rifampicin is the most potent PXR activator in this group. Our experiments in the detection of rifampicin using the nuclear receptor, PXR, showed good measurement reproducibility in the same day tested via three replicate consecutive injections of $1.0 \mu \mathrm{M}$ solution (see Figure 2 inset). Coefficients of variation (CVs) for intra-day measurements using a given system of MC and molecular-recognition phase are generally $10 \%$ or better $[16,18-20,23]$. Although for MCs that are nanostructured and biofunctionalized on different days the CVs can be considerably larger therefore calibration is necessary for each individual system. The relatively slow response kinetics is comparable to prior biosensor MC experiments [16,20,23]. This provides evidence that the ligands interact with the LBD causing relatively slow conformational changes in the immobilized PXR, which translates into a large apparent surface stress on the cantilever. The high binding affinity of human PXR for rifampicin followed by PCN has been observed by other researchers [7,9,15,28,40,41]. Although, 3-MC is predicted to have a low binding energy to the LBD of human PXR [42], it has been shown to be activated by members of the cytochrome P450 family of enzymes, which include activating CYP1A1/2 enzymes [42-45].

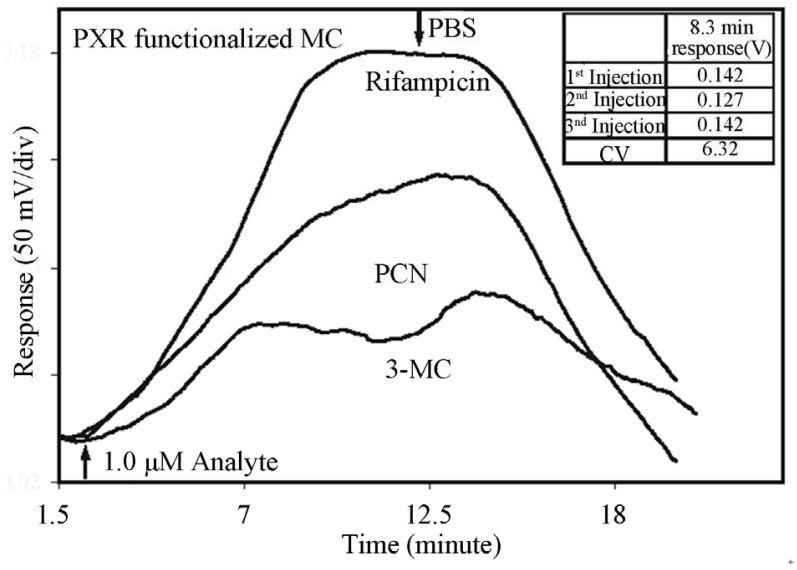

Figure 2. Comparison of nanomechanical responses of PXR functionalized MC on exposure to $1.0 \mu \mathrm{M}$ of rifampicin, $\mathrm{PCN}$, and 3-MC in PBS. Inset shows data for triplicate sequential injections of $1.0 \mu \mathrm{M}$ rifampicin with a $\mathrm{CV}$ of 6.32 .

The magnitude response order of rifampicin $>$ PCN $>$ 3-MC is what we predicted from the literature. In our previous studies, reversible responses like those seen in Figure 2 are also observed for other bioreceptor functionalized dealloyed surfaces $[20,23]$.

Figure 3 compares the response of specific protein (PXR) functionalized MC to nonspecific protein (ovalbumin) functionalized MC (blank) on exposure to the same concentration $(1.0 \mu \mathrm{M})$ of rifampicin in $10 \mathrm{mM}$ PBS, pH 7.0. A large compressive response was observed due to the binding of rifampicin with a MC modified with PXR receptor whereas no response was observed when the same analyte was exposed to the nonbinding protein (ovalbumin) immobilized MC. It is important to note that our system does not show a nonspecific blank response. This indicates that the surface immobilization procedure does not significantly alter the PXR's LBD function and that the observed responses represent specific interactions. However, it can not be assumed that the surface immobilized receptors will retain the same ligand binding affinity as observed in free form. Figure 3 further compares the specific and nonspecific responses to $1.0 \mu \mathrm{M}$ rifampicin, PCN, and 3-MC to immobilized PXR and ovalbumin (blank).

EDC exposure can be adverse even at very small concentrations [46]. EDCs can alter or inhibit the function of the endocrine system by binding to estrogen receptors, which are part of the nuclear receptor superfamily [4749]. Studies have shown that the nuclear receptor PXR binds to certain EDCs with different affinities [50,51]. Figure 4 shows the nanomechanical response magnitude of PXR functionalized MCs on exposure to $1.0 \mu \mathrm{M}$ EDCs, phthalic acid, nonylphenol, and bisphenol A (in $10 \mathrm{mM}$ PBS, $\mathrm{pH}$ 7.0). The response magnitude order of 


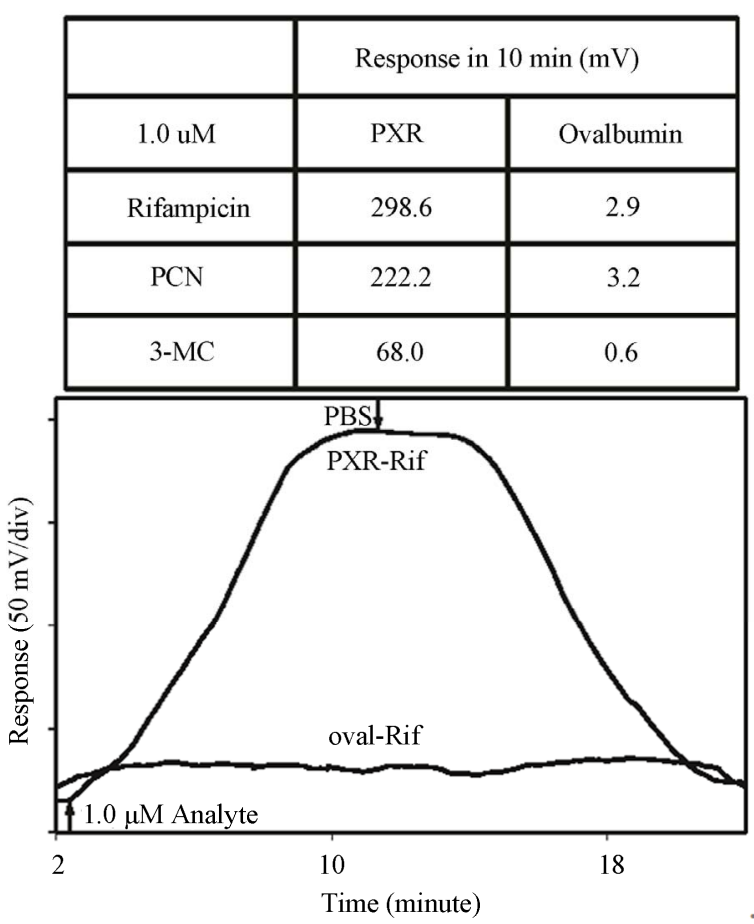

Figure 3. Table compares the nanomechanical responses of PXR and ovalbumin functionalized MCs exposed to $1.0 \mu \mathrm{M}$ rifampicin, PCN, and 3-MC in PBS. Representative traces of ovalbumin and PXR functionalized MCs on exposure to $1.0 \mu \mathrm{M}$ rifampicin are shown.

PXR with these three EDCs is similar to prior work with phthalic acid > nonylphenol and with relatively no response or a very low response to bisphenol A [50]. After MC exposure to EDCs, we injected $1.0 \mu \mathrm{M}$ rifampicin, a potent PXR activator, for comparison. As predicted, the

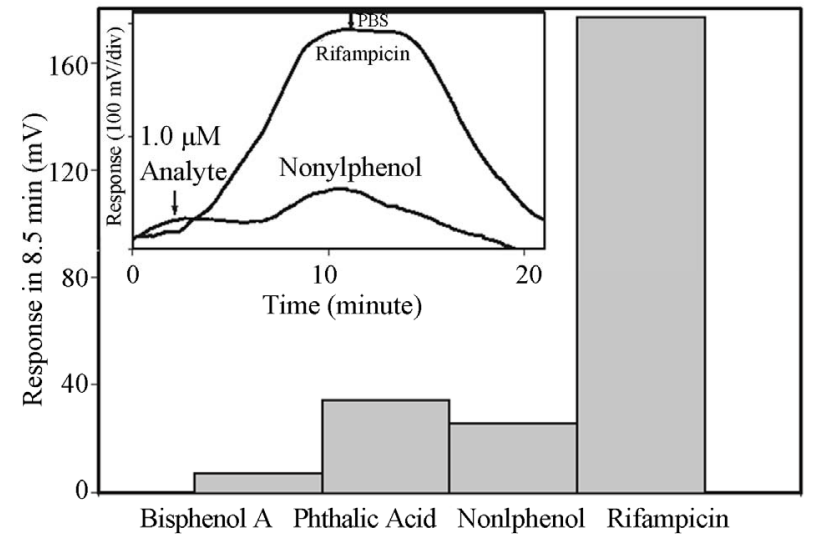

Figure 4. Comparison of response magnitudes in $8.5 \mathrm{~min}-$ utes of PXR functionalized MC on exposure to $1.0 \mu \mathrm{M}$ analytes in PBS, which include three EDCs. The inset shows representative responses of PXR functionalized MC exposed to $1.0 \mu \mathrm{M}$ nonylphenol and rifampicin. rifampicin caused a large compressive response when compared to the EDCs (see inset).

\subsection{Calibration and Sequential Exposure Studies}

In Figure 5 the kinetic response of the PXR functionalized cantilever at 7 minutes of exposure is plotted against different concentrations of rifampicin in the range of 0.1 $\mathrm{nM}$ to $1 \mu \mathrm{M}$ where the response increases with increasing concentration. The response magnitude does not increase as significantly as predicted with increasing concentration at relatively high concentrations. This may be due in part to the structural flexibility of the LBD. The expansion of the LBD size to accommodate for rifampicin's large size could have an effect on the interaction's translation to MC surface stress. Rifampicin is one of the largest known ligands for PXR at 823 Da [14]. As illustrated in the figure, the response increased predictably, but reached a saturation plateau by $100 \mathrm{nM}$. The inset in Figure 5 shows a linear dynamic range for two orders of magnitude (the first data point corresponds to the lowest concentration in Figure 5 of $0.1 \mathrm{nM}$ ) in concentration.

The renaturing of the protein after ligand exposure may influence receptor calibration experiments, thus sequential ligand exposure was studied. Immobilized LBD PXR was exposed to sequential injections of $1.0 \mu \mathrm{M}$ rifampicin in $10 \mathrm{mM}$ PBS, pH 7.0 with varying exposure and recovery time. The injection times and recovery times were approximately the same. As injection time increased the response maximum increased as well, which is likely a result of increased exposure time. This longer interaction time may provide larger kinetic responses, which is the nanomechanical measured parameter. All injection volumes and times were considerably less than saturation and were reversible, although baseline was not always reached with shorter recovery times. Exposure and recovery times of LBD PXR were also

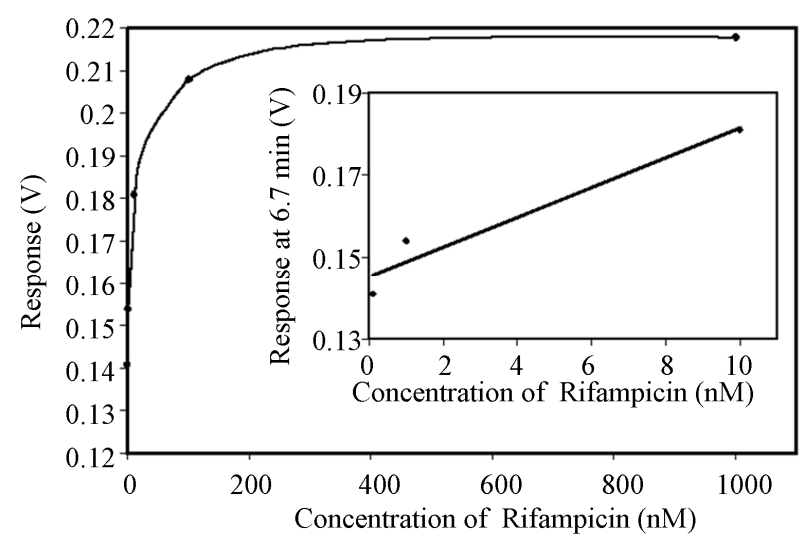

Figure 5. Responses to rifampicin at 6.7 minutes for PXR are plotted over a concentration range from $0.1 \mathrm{nM}$ to 1.0 $\mathrm{M}$, respectively. 
studied with two sequential three minute rifampicin injections then a 60 minute recovery time (background flow) then two more consecutive injections (three minutes and nine minutes). The first two injections had similar response maximums, $35.8 \mathrm{mV}$ versus $33.4 \mathrm{mV}$, although between injections baseline was not reached during the short recovery window. After a 60 minute recovery time or background flow, the three minute response to rifampicin increased to $58.3 \mathrm{mV}$. This was subsequently followed by a nine minute rifampicin injection where the response maximum almost doubled. This study may indicate that recovery time, exposure time, and analyte volume play a role in kinetic response maximums for the LBD PXR- rifampicin system.

\subsection{Effect of Different Molecular Tags on Measuring Surface Stress}

In our current functionalization procedure, the protein is immobilized on the MC surface with GA without appreciable denaturing [52]. This is accomplished by the aldehyde groups in the GA binding to the lysine residues in the receptor [53]. This makes the number and location of the lysine residues that make up the protein or receptor important to our random immobilization process. The tags on the LBD PXR allows for the purification of the receptor. The amino acids present in the tag and their location could be beneficial or detrimental to the nanomechanical responses of PXR functionalized MCs. GST-tagged human LBD PXR from Invitrogen has a calculated molecular mass of approximately $65 \mathrm{kDa}$. Human LBD PXR receptor is approximately $37 \mathrm{kDa}$, which leads to the calculated molecular mass for the GST-tag to be approximately $27 \mathrm{kDa}$. The GST-tag and the LBD PXR have similar molecular masses in this case. The 6-HIS-tag on the Astra Zeneca LBD PXR is approximately $2524 \mathrm{Da}$ and contains no lysine residues. Conversely, the GST-tag contains an estimated 21 lysine residues, depending on which version of the GST was used in the preparation. Thus, the 6-HIS-tagged LBD PXR was not bound to the surface by the tag, but by the nuclear receptor itself, where as the much larger GST-tag could have been the surface bound component.

Figure 6 compares the responses of 6-HIS-tagged LBD PXR and GST-tagged LBD PXR upon exposure to $1.0 \mu \mathrm{M}$ rifampicin in $10 \mathrm{mM}$ PBS, $\mathrm{pH}$ 7.0. The 6-HIS-tagged LBD PXR gives a large compressive response whereas the GST-tagged LBD PXR shows no response. This indicates that the GST-tag may preferentially bind to the surface leaving the PXR too far from the surface to produce nanomechanical surface stress. In prior work, random versus orientated antibody immobilization methods were studied, which demonstrated that

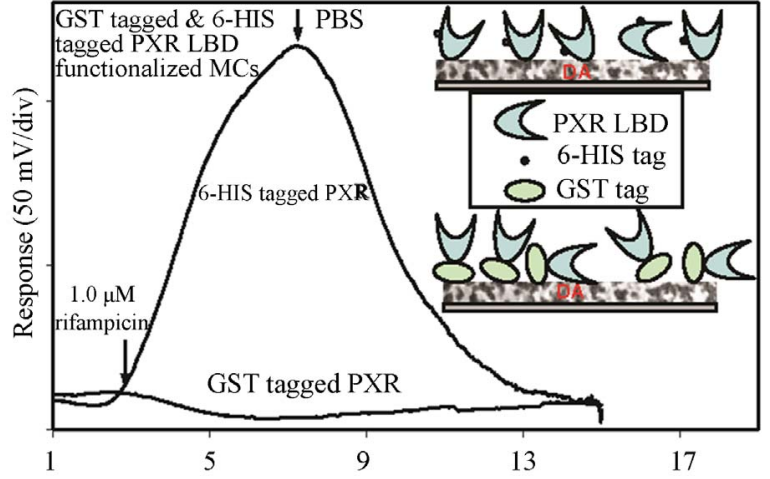

Figure 6. Comparison of 6-HIS-tagged PXR LBD and GSTtagged PXR LBD on exposure to 1.0 $\mu \mathrm{M}$ rifampicin in PBS. Inset shows a schematic depiction of 6-HIS tagged PXR and GST tagged PXR immobilized on DA MC surface, which highlights the spatial arrangement between the DA and the PXR LBD depending on the tag.

orientated functionalization did not provide higher responses as expected. Orientated anti-IgG and IgG immobilization was accomplished by using protein $\mathrm{A}$ as an orientation linker. This linker may have caused the antibody-antigen interaction not to be as readily translated into MC surface stress as the randomly orientated antibody [17]. As with the GST-tagged PXR case this may not allow the conformational change upon ligand binding to translate efficiently to measureable surface stress. The very small, lysine deficient 6-HIS-tag requires the receptor to be surface bound and could easily transfer conformational change to the MC surface although only some ligand binding sites may be accessible. This is illustrated through a schematic in Figure 6. Although in some instances the immobilized orientation of LBD PXR may block the ligand binding pocket thereby hindering ligand binding.

\subsection{LBD PXR and ER- $\alpha$ Array Study}

A single MC array functionalized with multiple biomolecular recognition phases will mimic a living system and it's interaction with contaminates and/or pharmaceuticals. EDCs or pharmaceuticals can stimulate changes in multiple receptor proteins. Therefore, having a MC array platform functionalized with a multiple receptor protein system (Figure 7) can provide information on total biological systems. MC arrays functionalized with different receptor proteins can simultaneously probe these living systems, which make them highly desirable analytical tools. LBD PXR and ER- $\alpha$ were differentially immobilized onto separate levers of one MC chip creating an array. Figure 7(a) shows a fluorescent microscope image of immobilized fluorescently labeled antibodies on sepa- 
rate levers prepared in the same manner as the LBD PXR and ER- $\alpha$ array. The receptor functionalized levers were exposed to $1.0 \mu \mathrm{M}$ rifampicin and the bending response was recorded. First, the LBD PXR functionalized lever was monitored with a five minute rifampicin injection, subsequently the ER- $\alpha$ immobilized lever was monitored in the same manner.

Figure 7(b) shows the response profiles of the LBD PXR and the ER- $\alpha$ when exposed to rifampicin in 10 $\mathrm{mM}$ PBS, pH 7.0. ER- $\alpha$ demonstrating a larger response than LBD PXR may be due to the structure of the receptors that are present. Immobilized ER- $\alpha$ contains both the

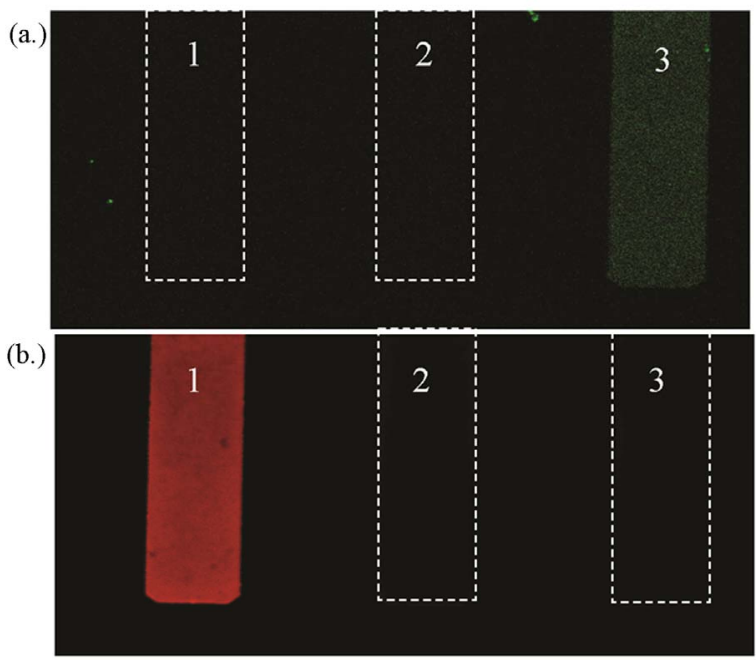

(a)

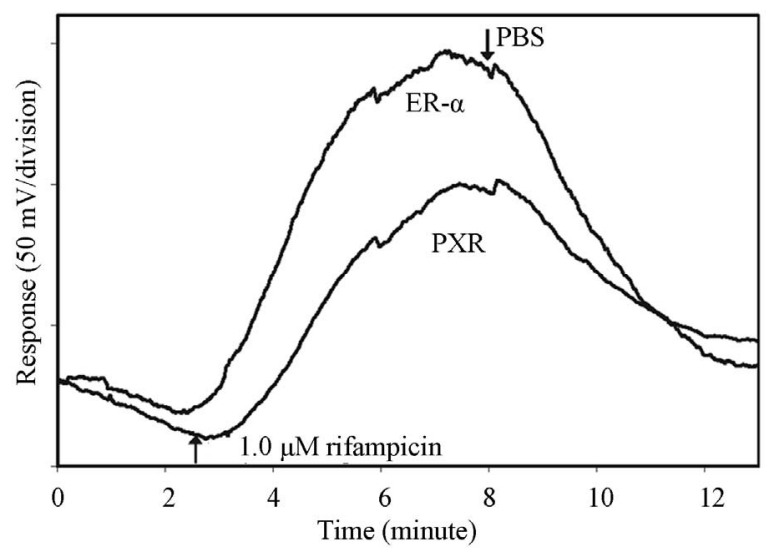

(b)

Figure 7. (a) Fluorescence microscopy images of differentially functionalized MCs: (a.) and (b.) are the same lever set where lever 1 is functionalized with Alexa Fluor 633 anti-IgG, lever 2 is not functionalized with antibody, lever 3 is functionalized with FITCanti-IgG. (a.) FITC-anti-IgG on lever 3 is excited by $488 \mathrm{~nm}$ laser. (b.) Alexa Fluor anti-IgG on lever 1 is excited by $633 \mathrm{~nm}$ laser. (b) MC array of PXR and ER- $\alpha$ exposed to $1.0 \mu \mathrm{M}$ rifampicin.
DNA binding domain and the LBD, whereas the immbilized PXR only contains the LBD and the small 6-HIStag. In previous studies with ER proteins, immobilization at the DNA binding sites which change configuration in response to conformational LBD changes may have been a factor in producing large surface stress responses [16]. In this case the ER- $\alpha$ interacts to some degree with rifampicin causing configuration change of the LBD, which may be translated into a conformational change in the DNA binding domain. This conformational change is transferred to surface stress on the MC. Another possibility for smaller PXR responses may be that it does not have time to fully unfold to accommodate for the large size of rifampicin, so the interaction between the receptor and analyte is limited, which hinders the response magnitude. In any event a synergistic system of receptors is demonstrated on a single MC array and there is evidence of endocrine disrupting behavior of rifampicin.

\section{Conclusions}

In summary, a sensitive, selective biosensor for the study of PXR activating chemicals has been developed using nanostructured MCs. Our results indicate that the interaction of LBD PXR with different ligands produced different cantilever responses showing the maximum response for the antibiotic rifampicin. PXR functionalized MCs showed responses to rifampicin in concentrations down to $0.1 \mathrm{nM}$. Dissimilarity in tag size and residue makeup also indicates differences in receptor-ligand binding translation into MC surface stress. The nuclear receptor array provides information about the interaction of rifampicin with both human PXR and ER- $\alpha$.

\section{Acknowledgements}

This research has been supported in part by a grant from the U.S. Environmental Protection Agency's Science to Achieve Results (STAR) program (EPA-83274001). The authors extend their gratitude to Dr. Melanie Eldridge of the Center of Biotechnology at the University of Tennessee, Knoxville for her useful discussions and to Dr. Roderic Cole for his assistance in obtaining PXR.

\section{REFERENCES}

[1] D. R. Nelson, L. Koymans, T. Kamataki, J. J. Stegeman, R. Feyereisen, D. J. Waxman, M. R. Waterman, O. Gotoh, M. J. Coon, R. W. Estabrook, I. C. Gunsalus and D. W. Nebert, "P450 Superfamily: Update on New Sequences, Gene Mapping, Accession Numbers and Nomenclature,” Pharmacogenetics, Vol. 6, 1996, pp. 1-42. doi:10.1097/00008571-199602000-00002

[2] S. A. Kliewer, J. M. Lehmann and T. M. Willson, “Orphan Nuclear Receptors: Shifting Endocrinology into Reverse,” Science, Vol. 284, 1999, pp. 757-760. doi:10.1126/science.284.5415.757 
[3] E. L. Michalets, "Update: Clinically Significant Cyto-Chrome P-450 Drug Interactions,” Pharmacotherapy, Vol. 18, 1998, pp. 84-112.

[4] P. R. Ortiz de Montellano, "Cytochrome P450: Structure, Mechanism and Biochemistry,” Plenum Publishers, New York, 2005, pp. 377-425.

[5] J. Zhang, P. Kuehl, E. D. Green, J. W. Touchman, P. B. Watkins, A. Daly, S. D. Hall, P. Maurel, M. Relling, C. Brimer, K. Yasuda, S. A. Wrighton, M. Hancock, R. B. Kim, S. Strom, K. Thummel, C. G. Russell, J. R. Hudson, Jr., E. G. Schuetz and M. S. Boguski, "The Human Pregnane X Receptor: Genomic Structure and Identification and Functional Characterization of Natural Allelic Variants,” Pharmacogenetics, Vol. 11, 2001, pp. 555-572. doi:10.1097/00008571-200110000-00003

[6] S. A. Kliewer, J. T. Moore, L. Wade, J. L. Staudinger, M. A. Watson, S. A. Jones, D. D. McKee, B. B. Oliver, T. M. Willson, R. H. Zetterström, T. Perlmann and J. M. Lehmann, “An Orphan Nuclear Receptor Activated by Pregnanes Defines a Novel Steroid Signaling Pathway,” Cell, Vol. 92, 1998, pp. 73-82. doi:10.1016/S0092-8674(00)80900-9

[7] B. Blumberg, W. Sabbagh, Jr., H. Juguilon, J. Bolado, Jr., C. M. van Meter, E. S. Ong and R. M. Evans, "SXR, a Novel Steroid and Xenobiotic-Sensing Nuclear Receptor,” Genes and Development, Vol. 12, 1998, pp. 3195-3205. doi:10.1101/gad.12.20.3195

[8] G. Bertilsson, J. Heidrich, K. Svensson, M. Åsman, L. Jendeberg, M. Sydow-Bäckman, R. Ohlsson, H. Postlind, P. Blomquist and A. Berkenstam, "Identification of a Human Nuclear Receptor Defines a New Signaling Pathway for Cyp3a Induction," Proceedings of the National Academy of Sciences, Vol. 95, 1998, pp. 12208-12213. doi:10.1073/pnas.95.21.12208

[9] R. E. Watkins, G. B. Wisely, L. B. Moore, J. L. Collins, M. H. Lambert, S. P. Williams, T. M. Willson, S. A. Kliewer and M. R. Redinbo, "The Human Nuclear Xenobiotic Receptor PXR: Structural Determinants of Directed Promiscuity,” Science, Vol. 292, 2001, pp. 2329-2333. doi:10.1126/science.1060762

[10] L. B. Moore, J. M. Maglich, D. D. McKee, B. Wisely, T. M. Willson, S. A. Kliewer, M. H. Lambert and J. T. Moore, "Pregnane X Receptor (PXR), Constitutive Androstane Receptor (CAR) and Benzoate X Receptor (BXR) Define Three Pharmacologically Distinct Classes of Nuclear Receptors," Molecular Endocrinology, Vol. 16, 2002, pp. 977-986. doi:10.1210/me.16.5.977

[11] C. Zhou, S. Verma and B. Blumberg, "The Steroid and Xenobiotic Receptor (Sxr), Beyond Xenobiotic Metabolism,” Nuclear Receptor Signaling, Vol. 7, 2009, pp. 1-21.

[12] S. A. Kliewer, B. Goodwin and T. M. Willson, "The Nuclear Pregnane X Receptor: A Key Regulator of Xenobiotic Metabolism,” Endocrine Reviews, Vol. 23, 2002, pp. 687-702. doi:10.1210/er.2001-0038

[13] J. E. Chrencik, J. Orans, L. B. Moore, Y. Xue, L. Peng, J. L. Collins, G. B. Wisely, M. H. Lambert, S. A. Kliewer and M. R. Redinbo, "Structural Disorder in the Complex of Human Pregnane X Receptor and the Macrolide Antibiotic Rifampicin,” Molecular Endocrinology, Vol. 19, 2005, pp. 11251134. doi:10.1210/me.2004-0346
[14] J. T. Moore and S. A. Kliewer, 'Use of the Nuclear Recaptor Pxr to Predict Drug Interactions,” Toxicology, Vol. 153, 2000, pp. 1-10. doi:10.1016/S0300-483X(00)00300-0

[15] P. Dutta, K. Hill, P. G. Datskos and M. J. Sepaniak, "Development of a Nanomechanical Biosensor for Analysis of Endocrine Disrupting Chemicals," Lab Chip, Vol. 7, 2007, pp. 1184-1191. doi:10.1039/b704723a

[16] K. Hill, P. Dutta, A. Zareba, M. L. Eldridge and M. J. Sepaniak, "Morphological and Chemical Optimization of Microcantilever Surfaces for Thyroid System Sensing and Beyond," Analytica Chimica Acta, Vol. 625, 2008, pp. 55-62. doi:10.1016/j.aca.2008.07.005

[17] C. A. Tipple, N. V. Lavrik, M. Culha, J. Headrick, P. Datskos and M. J. Sepaniak, "Nanostructured Microcantilevers with Functionalized Cyclodextrin Receptor Phases: Self Assembled Monolayers and Vapor Deposited Films," Analytical Chemistry, Vol. 74, 2002, pp. 3118-3126. doi:10.1021/ac020074o

[18] P. Dutta, P. J. Chapman, P. G. Datskos and M. J. Sepaniak, "Characterization of Ligand-Functionalized Microcantilevers for Metal Ion Sensing,” Analytical Chemistry, Vol. 77, 2005, pp. 6601-6608. doi:10.1021/ac051082i

[19] P. Dutta, J. Sanseverino, P. G. Datskos and M. J. Sepaniak, "Detection of Cytokine Using a Microcantilever Biosensor," NanoBiotechnology, 1, 2005, pp. 237-244. doi:10.1385/NBT:1:3:237

[20] R. Berger, E. Delamarche, H. P. Lang, C. Gerber, J. K. Gimzewski, E. Meyer and H. J. Guntherodt, "Surface Stress in the Self-Assembly of Alkanethiols on Gold,” Science, Vol. 276, 1997, pp. 2021-2024. doi:10.1126/science.276.5321.2021

[21] T. Thundat, E. A. Wachter, S. L. Sharp and R. J. Warmack, "Detection of Mercury Vapor Using Resonating Microcantilevers,” Applied Physics Letters, Vol. 66, 1995, pp. 1695-1697. doi:10.1063/1.113896

[22] P. Dutta, C. A. Tipple, N. V. Lavrik, P. G. Datskos, O. Hofstetter and M. J. Sepaniak, "Enantioselective Sensors Based on Antibody Mediated Nanomechanics,” Analytical Chemistry, Vol. 75, 2003, pp. 2342-2348. doi:10.1021/ac034031z

[23] G. Wu, R. H. Datar, K. M. Hansen, T. Thundat, R. J. Cote and A. Majumdar, "Bioassay of Prostate-Specific Antigen (Psa) Using Microcantilevers," National Biotechnology, Vol. 19, 2001, pp. 856-860. doi:10.1038/nbt0901-856

[24] C. Grogan, R. Raiteri, G. M. O’Connor, T. J. Glynn, V. Cunningham, M. Kane, M. Charlton and D. Leech, "Characterization of an Antibody Coated Microcantilever as a Potential Immuno-Based Biosensor,” Biosensor Bioelectronics, Vol. 17, 2002, pp. 201-207. doi:10.1016/S0956-5663(01)00276-7

[25] J. Fritz, M. K. Baller, H. P. Lang, H. Rothuizen, P. Vettiger, E. Meyer, H. Guntherodt, C. Gerber and J. K. Gimzewski, "Translating Biomolecular Recognition into Nanomechanics," Science, Vol. 288, 2000, pp. 316-318. doi:10.1126/science.288.5464.316

[26] G. Shekhawat, S. H. Tark and V. P. Dravid, "MOSFET-Embedded Microcantilevers for Measuring Deflection in Biomolecular Sensors,” Science, Vol. 311, 2006, pp. 15921595. doi:10.1126/science. 1122588 
[27] S. A. Jones, L. B. Moore, J. L. Shenk, G. B. Wisely, G. A. Hamilton, .D. D. McKee, N. C. O. Tomkinson, E. L. LeCluyse, M. H. Lambert, T. M. Willson, S. A. Kliewer and J. T. Moore, "The Pregnane X Receptor: A Promis-Cuous Xenobiotic Receptor That Has Diverged during Evolution,” Molecular Endocrinology, Vol. 14, 2000, pp. 27-39. doi:10.1210/me.14.1.27

[28] S. J. Shukla, D. Nguyen, R. MacArthur, A. Simeonov, W. J. Frazee, T. M. Hallis, B. D. Marks, U. Singh, H. C. Eliason, J. Printen, C. P. Austin, J. Inglese and D. S. Auld, "Identification of Pregnane X Receptor Ligands Using Time-Resolved Fluorescence Resonance Energy Transfer and Quantitative HighThroughput Screening," Assay and Drug Development Technologies, Vol. 7, 2009, pp. 143-169.

doi:10.1089/adt.2009.193

[29] G. G. Stoney, "The Tension of Metallic Films Deposited by Electrolysis," Proceeding of the Royal Society of London, Serial A, Vol. 82, 1909, pp. 172-177.

[30] N. P. Mahalik, "Micromanufacturing and Nanotechnology," Springer, Berlin, 2006, pp. 299-321.

[31] N. V. Lavrik, C. A. Tipple, M. J. Sepaniak and P. G. Datskos, "Enhanced Chemi-Mechanical Transduction at Nanostructured Interfaces,” Chemical Physics Letters, Vol. 336, 2001, pp. 371-376. doi:10.1016/S0009-2614(01)00155-5

[32] J. R. Premkumar, O. Lev, R. S. Marks, B. Polyak, R. Rosen and S. Belkin, "Antibody-Based Immobilization of Bioluminescent Bacterial Sensor Cells,” Talanta, Vol. 55, 2001, pp. 1029-1038. doi:10.1016/S0039-9140(01)00533-1

[33] S. W. Park, Y. I. Kim, K. H. Chung, S. I. Hong and S. W. Kim, "Covalent Immobilization of Gl-7-Aca Acylase on Silica Gel Through Silanization,” Reactive Functional Polymers, Vol. 51, 2002, pp. 79-92. doi:10.1016/S1381-5148(02)00028-7

[34] P. J. Chapman, Z. Long, P. G. Datskos, R. Archibald and M. J. Sepaniak, "Ligand-Functionalized Microcantilever Arrays for Metal Ion Sensing,” Analytical Chemistry, Vol. 79, 2007, pp. 7062-7068. doi:10.1021/ac070754x

[35] L. R. Senesac, P. Dutta, P. G. Datskos and M. J. Sepaniak, "Analyte Identification Using Differentially-Functionalized Microcantilever Arrays and Artificial Neural Nets,” Analytical Chemistry Acta, Vol. 558, 2006, pp. 94-101. doi:10.1016/j.aca.2005.11.024

[36] P. J. Chapman, Z. Long, P.G. Datskos, R. Archbald and M. J. Sepaniak, "Ligand-Functionalized Microcantilever Arrays for Metal Ion Sensing,” Analytical Chemistry, Vol. 79, 2007, pp. 7062-7068. doi:10.1021/ac070754x

[37] Z. Long, J. Story, S. Lewis and M. J. Sepaniak "Landfill Siloxane Gas Sensing Using Differentiating, Responsive Phase Coated Microcantilever Arrays” Analytical Chemistry, Vol. 81, 2009, pp. 2575-2580. doi:10.1021/ac802494v

[38] M. J. Sepaniak, P. G. Datskos, N. V. Lavrik and C. Tipple, "Microcantilever Transducers: A New Approach in Sensor Technology,” Analytical Chemistry, Vol. 74, 2002, pp. 568A575A.

[39] J. L. Staudinger, B. Goodwin, S. A. Jones, D. Hawkins-Brown, K. I. MacKenzie, A. LaTour, Y. Liu, C. D. Klaassen, K. K. Brown, J. Reinhard, T. M. Willson, B. H. Koller and S. A. Kliewer, "The Nuclear Receptor PXR is a Lithocholic Acid
Sensor That Protects against Liver Toxicity," Processings of the National Academy Sciences, Vol. 98, 2001, pp. 3369-3374. doi:10.1073/pnas.051551698

[40] E. Y. H. Yeung, T. Sueyoshi, M. Negishi and T. K. H. Chang, "Identification of Ginkgo Biloba as a Novel Activator of Pregnane X Receptor,” Drug Metabolism and Disposition, Vol. 36, 2008, pp. 2270-2276. doi:10.1124/dmd.108.023499

[41] S. Kortagere, D. Chekmarev, W. J. Welsh and S. Ekins, "Hybrid Scoring and Classification Approaches to Predict Human Pregnane X Receptor Activators,” Pharmaceutical Research, Vol. 26, 2009, pp. 1001-1011. doi:10.1007/s11095-008-9809-7

[42] P. Li, "Evaluation of Luciferin-Isopropyl Acetal as a CYP3A4 Substrate for Human Hepatocytes: Effects of Organics Solvents, Cytochrome P450 (P450) Inhibitors and P450 Inducers,” Drug Metabolism and Disposition, Vol. 37, 2009, pp. 1598-1603. doi:10.1124/dmd.109.027268

[43] M. Abdelrahim, E. Ariazi, K. Kim, S. Khan, R. Barhoumi, R. Burghardt, S. Liu, D. Hill, R. Finnell, B. Wlodarczyk, V. C. Jordan and S. Safe, "3-Methylcholanthrene and Other Aryl Hydrocarbon Receptor Agonists Directly Activate Estrogen Receptor,” Cancer Research, Vol. 66, 2006, pp. 2459-2467. doi:10.1158/0008-5472.CAN-05-3132

[44] J. P. Jackson, K. K. Kabirov, I. M. Kapetanovic and A. Lyubimov, "In Vitro Assessment of p450 Induction Potential of Novel Chemopreventive Agents sr13668, 9-cis-uab30 and Pentamethychromanol In Primary Cultures of Human Hepatocytes,” Chemical-Biology Interactive, Vol. 179, 2009, pp. 263-272. doi:10.1016/j.cbi.2008.12.005

[45] H. Kuramitz, J. Natsui, K. Sugawara, S. Itoh and S. Tanaka, "Electrochemical Evaluation of the Interaction between Endocrine Disruptor Chemicals and Estrogen Receptor Using 17-Estradiol Labeled with Daunomycin,” Analytical Chemistry, Vol. 74, 2002, pp. 533-538. doi:10.1021/ac010426b

[46] G. G. J. M. Kuiper, J. G. Lemmen, B. Carlson, J. C. Corton, S. H. Safe, P. T. Van Der Saag, B. Van Der Burg and J. Gustafsson, "Interaction of Estrogenic Chemicals and Phytoestrogens with Estrogen Receptor,” Endocrinology, Vol. 139, 1998, pp. 4252-4263. doi:10.1210/en.139.10.4252

[47] S. Stoica, B. S. Katzenellenbogen and M. B. Martin, “Activation of Estrogen Receptor-by the Heavy Metal Cadmium,” Molecular Endocrinology, Vol. 14, 2000, pp. 545-553. doi:10.1210/me.14.4.545

[48] D. J. Mangelsdorf, C. Thummel, M. Besto, P. Herrlich, G. Schütz, K. Umesono, B. Blumberg, P. Kastner, M. Mark, P. Chambon and R. M. Evans, "The Nuclear Receptor Superfamily: The Second Decade,” Cell, Vol. 83, 1995, pp. 835-839. doi:10.1016/0092-8674(95)90199-X

[49] H. Masuyama, Y. Hiramatsu, M. Kunitomi, T. Kudo and P. N. MacDonald, "Endocrine Disrupting Chemicals, Phthalic Acid and Nonylphenol, Activate Pregnane X Receptor-Mediated Transcription,” Molecular Endocrinology, Vol. 14, 2000, pp. 421-428. doi:10.1210/me.14.3.421

[50] H. Masuyama, N. Suwaki, Y. Tateishi, H. Nakatsukasa, T. Seqawa and Y. Hiramatsu, "The Pregnane X Receptor Regulates Gene Expression in a Ligand and Promoter-Selective 
Fashion,” Molecular Endocrinology, Vol. 19, 2005, pp. 11701180. doi:10.1210/me.2004-0434

[51] D. Hopwood, C. R. Allen and M. McCabe, "The Reactions between Glutaraldehyde and Various Protein. An Investigation of Their Kinetics,” Histochemical Journal, Vol. 2, 1970, pp.
137-150. doi:10.1007/BF01003541

[52] D. R. Walt and V. I. Agayn, "The Chemistry of Enzyme and Protein Immobilization with Glutaraldehyde,” Trends Analytical Chemistry, Vol. 13, 1994, pp. 425-430. doi:10.1016/0165-9936(94)85023-2 